\title{
Primary hyperparathyroidism caused by mediastinal ectopic parathyroid adenoma
}

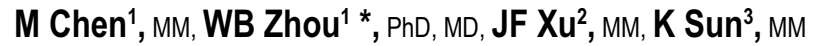

Departments of ${ }^{1}$ Endocrinology, ${ }^{2}$ Radiology, and ${ }^{3}$ Pathology, The First Affiliated Hospital, College of Medicine, Zhejiang University, \#79, Qingchun Road, Hangzhou, Zhejiang, 310003, China

Hong Kong Med J 2017;23:411-3

DOI: $10.12809 / \mathrm{hkmj} 164957$

\section{Case report}

A 37-year-old Chinese woman was admitted to the First Affiliated Hospital, College of Medicine, Zhejiang University, China in July 2009 with pain in the upper back and hip for 9 months. She denied any chronic medication or illness. Her serum calcium level was $2.73 \mathrm{mmol} / \mathrm{L}$ (reference range [RR], 2.08$2.60 \mathrm{mmol} / \mathrm{L}$ ), phosphorus $0.68 \mathrm{mmol} / \mathrm{L}$ (RR, 0.81$1.62 \mathrm{mmol} / \mathrm{L}$ ), alkaline phosphatase $366 \mathrm{IU} / \mathrm{L}$ (RR, 30-115IU/L), and intact parathyroid hormone (iPTH) $1154 \mathrm{pg} / \mathrm{mL}$ (RR, $12-65 \mathrm{pg} / \mathrm{mL}$ ). T-score and Z-score for femoral bone mineral density were -2.7 and -2.8 , respectively. Cervical computed tomography (CT) scan and thyroid, parathyroid, and abdominal (including pancreas, adrenals) ultrasonography were unremarkable. Anterior planar technetium (99mTc) sestamibi (MIBI) scintigraphy images of the neck and chest showed a focal shadow with intense tracer uptake in the superior thorax at 15 minutes (Fig a) and 120 minutes (Fig b) following injection of ${ }^{99} \mathrm{~m}$ Tc-MIBI. Thoracic CT revealed a contrastenhanced nodule with soft tissue density of $2.9 \times 1.3$ $\mathrm{cm}$ in the anterior mediastinum (Fig c). Thoracic and abdominal CT showed a brown tumour in the right omoplate, the fourth posterior left rib, and iliac bone (Figs d and e).

A mid-sternal thoracotomy was performed. A dark red mass of $4 \times 2.4 \times 1 \mathrm{~cm}$ was found in the
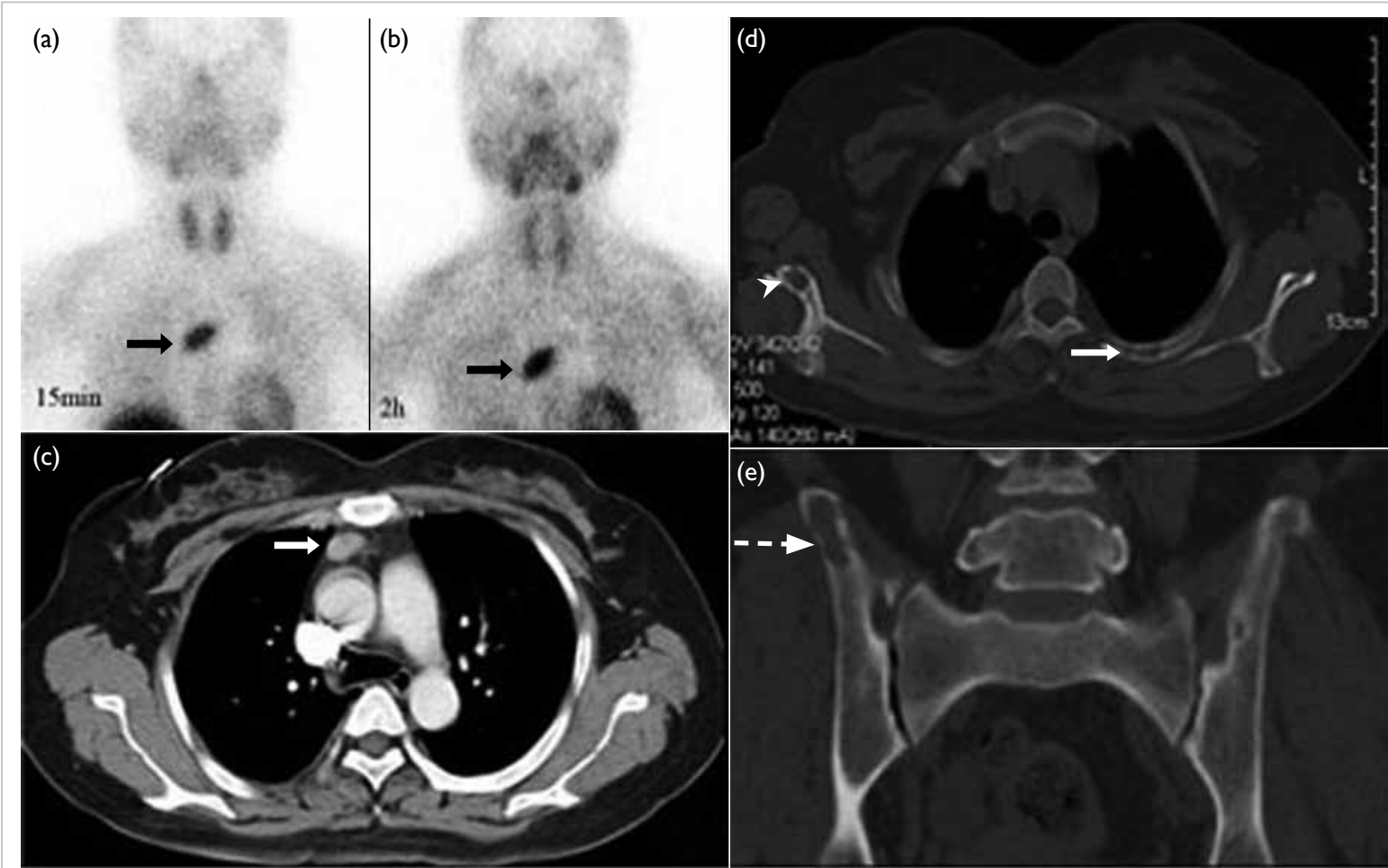

FIG. Technetium ( ${ }^{99 \mathrm{~m}} \mathrm{Tc}$ ) sestamibi showing (a) tracer uptake by thyroid glands and the gland in the superior thorax at 15 minutes (arrow); (b) persistent activity in the superior mediastinum at 2 hours (arrow). (c) Contrast-enhanced computed tomography (CT) demonstrating a nodular shadow with soft tissue density $(2.9 \times 1.3 \mathrm{~cm}$, clear borders) in the anterior mediastinum (arrow). (d) Thoracic and (e) abdominal CT showing brown tumour in the right omoplate (arrowhead), left fourth posterior rib (arrow), and iliac bone (dash arrow) 
thymus isthmus and resected. The mediastinal mass was covered with a thin fibrous capsule and on section showed a greyish cut surface. Histology confirmed the presence of ectopic parathyroid adenoma composed predominantly of oxyphil cells arranged in acinar pattern. Serum calcium level was $2.18 \mathrm{mmol} / \mathrm{L}$ and $\mathrm{iPTH} 17.2 \mathrm{pg} / \mathrm{mL} 16$ hours after surgery. Hypocalcaemia (serum calcium, 1.68 $\mathrm{mmol} / \mathrm{L}$ ) and hungry bone syndrome occurred 3 days after surgery and gradually improved over a week with calcium carbonate and calcitriol supplementation that was started after surgery and continued thereafter. Serum calcium and iPTH remained normal after 5 years' follow-up.

\section{Discussion}

A diagnosis of primary hyperparathyroidism in a symptomatic patient is made in the presence of hypercalcaemia, hypophosphataemia, and raised levels of alkaline phosphatase and iPTH, ${ }^{1}$ as demonstrated in our case. Primary hyperparathyroidism occurs in approximately $1 \%$ of the adult population, commonly due to solitary parathyroid adenomas (85\%). ${ }^{1}$ Primary hyperparathyroidism due to ectopic parathyroid adenomas can pose diagnostic and management challenges, especially when imaging studies provide limited sensitivity. ${ }^{2}$

The embryological origin of the parathyroid glands is the endoderm of the third and fourth pharyngeal pouches, from where these glands migrate to their usual position behind the thyroid gland. It is well known that parathyroid glands can be found in aberrant locations, mainly in the thyroid parenchyma or in the mediastinum. ${ }^{3}$ The high incidence of ectopic inferior parathyroid glands has been attributed to abnormal migration during embryogenesis. Since parathyroid glands lack capsular fixation, an ectopic parathyroid gland may also develop from a gland that is initially present in a normal anatomic position but which enlarges and is displaced to an ectopic location where there is little resistance.

Ultrasonography is commonly used to locate enlarged parathyroid glands due to its convenience and low cost. Its ability to detect abnormalities, however, depends on the experience and skill of the operator so sensitivity in the localisation of enlarged parathyroid glands varies greatly (44\%-87\%). ${ }^{2}$ Ectopic parathyroid adenomas may be detected with MIBI at a sensitivity level almost identical to that of orthotopic adenomas. Focal increased activity separated from the lower pole of the thyroid on MIBI images gives a high probability for locating ectopic parathyroid adenoma in the thymus. Of note, ${ }^{99 \mathrm{~m} T c-}$ MIBI is a non-specific tracer that is taken up by mitochondria so any mitochondria-rich cells may show uptake. Mitochondrial density in an adenoma is also a major factor that can cause prolonged retention of ${ }^{99 \mathrm{~m}} \mathrm{Tc}$. This is evidenced by the number of mitochondrion in lesions detected by scintigraphy that is significantly higher than in those that are missed, with the highest ratio of mitochondria per cell found in oxyphil cells. The typical pattern in a parathyroid adenoma demonstrates a prolonged retention of ${ }^{99 \mathrm{~m}} \mathrm{Tc}-\mathrm{MIBI}$ in the adenoma with rapid washout of the tracer from normal thyroid tissue. The degree of MIBI uptake in parathyroid adenomas has been reportedly correlated with the size of gland and the cytological composition (greater uptake seen in adenomas with dominance of oxyphil cells over chief cells). ${ }^{2}$

Of note, CT may further contribute to the identification of ectopic parathyroids and the differential diagnosis from other lesions. In our cases, the parathyroid glands were not located by ultrasonography although a combination of MIBI scintigraphy and CT imaging accurately localised the tumour in the anterior mediastinum. This highlights the usefulness of combining multiple imaging techniques to locate an ectopic active parathyroid gland. These combinations are cost-effective, and considered an approach to routine preoperative localisation of ectopic parathyroid adenomas, especially in cases with a negative MIBI scan. ${ }^{4}$

Hungry bone syndrome refers to the rapid, profound, and prolonged hypocalcaemia associated with hypophosphataemia following parathyroidectomy as a result of extensive remineralisation. ${ }^{5}$ Various risk factors of hungry bone syndrome include older age, large parathyroid adenoma, overt bone disease, and vitamin D deficiency. Consequently, transient hypocalcaemia is frequently encountered postoperatively; the presence of mild hypocalcaemia provides reassurance that the hyperactive adenomatous gland has been successfully removed.

For any hypercalcaemia and high level of PTH without parathyroid adenoma in the neck, physicians should remain alert and continue to search for ectopic locations using a combination of imaging techniques. The mediastinum must be cautiously explored since it is a very common location for ectopic parathyroid adenoma. The combination of several imaging techniques has an incremental effect on the localisation of ectopic parathyroid adenomas compared with use of either one technique alone.

\section{References}

1. AACE/AAES Task Force on Primary Hyperparathyroidism. The American Association of Clinical Endocrinologists and the American Association of Endocrine Surgeons position statement on the diagnosis and management of primary hyperparathyroidism. Endocr Pract 2005;11:4954.

2. Haber RS, Kim CK, Inabnet WB. Ultrasonography for preoperative localization of enlarged parathyroid glands 
in primary hyperparathyroidism: comparison with (99m) technetium sestamibi scintigraphy. Clin Endocrinol (Oxf) 2002;57:241-9.

3. Noussios G, Anagnostis P, Natsis K. Ectopic parathyroid glands and their anatomical, clinical and surgical implications. Exp Clin Endocrinol Diabetes 2012;120:60410.

4. Elaraj DM, Sippel RS, Lindsay S, et al. Are additional localization studies and referral indicated for patients with primary hyperparathyroidism who have negative sestamibi scan results? Arch Surg 2010;145:578-81.

5. Witteveen JE, van Thiel S, Romijn JA, Hamdy NA. Hungry bone syndrome: still a challenge in the post-operative management of primary hyperparathyroidism: a systematic review of the literature. Eur J Endocrinol 2013;168:R4553. 